\title{
A Novel Detection System Consisting of a Large-Area Sensor and a Multicell Si-Pad Array Operated in Spectroscopic Mode for X-Ray Breast Imaging
}

\author{
D. G. Darambara, Member, IEEE, P. J. Sellin, Member, IEEE, and G. Maehlum
}

\begin{abstract}
The ability of coherent X-ray scatter to provide the molecular structure of breast tissues could add a new dimension in X-ray breast imaging capable of tracking the molecular structural changes during disease progression and of improving the sensitivity to low-contrast lesions without increasing the radiation dose. Work is under way to build a laboratory prototype dual-sensor breast-imaging scanning system, which combines the diagnostic information from both the transmitted primary and the forward scattered $X$-rays. This required the design and development of a coherent $X$-ray scatter detection system based on a high-resistivity multielement two-dimensional (2-D) Si-pad array, a multichannel low-noise pulse processing front-end electronics chip, the XA1.3, and a new DAQ system. X-rays in the energy range of 17-45 keV can be detected with a FWHM energy resolution of 1-3 keV. Results on the characterization and optimization of the detector-readout electronics-DAQ system and its performance to measure diffraction signatures of most commonly used breast-equivalent materials of interest are presented.
\end{abstract}

Index Terms-Biomedical X-ray imaging, multi-element $\mathrm{Si}$ detector, X-ray breast imaging, X-ray coherent scattering, X-ray imaging detectors.

\section{INTRODUCTION}

D ESPITE significant advances, the major challenge in diagnostic X-ray imaging remains the determination of small size lesions in a low-contrast environment, while keeping the dose applied as low as possible. The screening situation in the diagnosis of breast cancer has not changed appreciably, and $\mathrm{X}$-ray mammography remains the gold standard for screening, although its specificity is $\sim 15 \%-20 \%$ [1]. In conventional $\mathrm{X}$-ray diagnostic imaging, the spatial distribution of the energy of the transmitted photons within the object is exploited to form the image of interest. Each spatial image coordinate carries information about the linear attenuation coefficient $\mu$ and the electron density. The subject of this research is to develop an alternative approach that allows the spatial distribution of $\mathrm{X}$-ray scattering properties, characterized by the differential scatter cross section $\mathrm{d} \sigma / \mathrm{d} \Omega$ to be mapped throughout the interior of the tissues concerned. The presence of diffraction maxima in the measured scattering profiles can thus be used to

Manuscript received November 15, 2003. This work was supported by the Wellcome Trust by Grant 062123 .

D. G. Darambara and P. J. Sellin are with the Physics Department, University of Surrey, Guildford, Surrey GU2 7XH, U.K. (e-mail: d.darambara@surrey.ac.uk; p.sellin@surrey.ac.uk).

G. Maehlum is with the IDE AS, N-1322 Høvik, Norway (e-mail: gunnar@ideas.no).

Digital Object Identifier 10.1109/TNS.2004.832578 provide enhanced tissue discrimination when compared to the conventional X-ray transmission imaging techniques.

The probability of the coherent X-ray scatter process increases with decreasing energy of photons and increasing number of scattering atoms. In medical X-ray imaging the coherent X-ray scatter peaks at precisely those energies where $\mathrm{X}$-ray breast imaging is performed [2], [3]. The low-angle $\left(\leq 10^{\circ}\right)$ behavior of coherent scatter is highly dependent on the chemical and structural properties of the scatterer. Its intensity, as a function of angle, is related to the molecular structure of the object being irradiated. Every molecular structure has its own signature scattering pattern. The origin of the peaks in the patterns is the interference of photons scattered from the molecules of the medium. The degree of interference is dependent on the momentum transfer $\chi$ given by $\chi=(1 / \lambda) \sin (\theta / 2)$, where $\theta$ is the scattering angle and $\lambda$ the wavelength of the X-rays. These interferences produce one or more peaks in intensity close to the forward direction and the complete distribution is considered as characteristic of the investigated sample. Each scattering profile represents the variation in differential scatter cross section as a function of the momentum transfer, which is determined by the incident energy and the angle of detection.

Many biological tissues have significant spatial ordering and readily diffract X-rays. X-ray diffraction measurements on biological materials [4]-[10] showed that the molecular structure of diseased tissues undergoes significant changes, and hence, the structure can be understood as a fingerprint of the disease. In particular, the X-ray coherent scatter cross sections of breast tissues are shown to be quite distinctive and different, in a material-specific way, for specific scattering angles and photon energies. The ability of coherent $\mathrm{X}$-ray scatter to provide the molecular structure of breast tissues could add a new dimension in $\mathrm{X}$-ray breast imaging capable of tracking the molecular structural changes during disease progression and of improving the sensitivity to low-contrast lesions without increasing the radiation dose.

The differential coherent scattering cross section can be expressed as: $\mathrm{d} \sigma_{\mathrm{coh}} / \mathrm{d} \Omega=\mathrm{r}_{0}^{2} / 2\left(1+\cos ^{2} \theta\right) \mathrm{F}_{\mathrm{m}}^{2}(\chi, \mathrm{Z})=$ $\left(\mathrm{d} \sigma_{\mathrm{T}} / \mathrm{d} \Omega\right) \mathrm{F}_{\mathrm{m}}^{2}(\chi, \mathrm{Z})$, where $\mathrm{d} \sigma_{\mathrm{T}} / \mathrm{d} \Omega$ is the Thomson differential cross section, $\mathrm{r}_{0}$ the classical electron radius, $\theta$ is the scattering angle, and $\mathrm{F}_{\mathrm{m}}(\chi, \mathrm{Z})$ is the molecular form factor. At large values of $\chi$, the molecular form factor approximates the independent atomic model (IAM) and is written as $\mathrm{F}_{\mathrm{m}}^{2}(\chi)=\sum \mathrm{n}_{\mathrm{i}} \mathrm{F}_{\mathrm{i}}^{2}\left(\chi, \mathrm{Z}_{\mathrm{i}}\right)$, where $\mathrm{n}_{\mathrm{i}}$ is the atomic abundance and $\mathrm{F}_{\mathrm{i}}^{2}\left(\chi, \mathrm{Z}_{\mathrm{i}}\right)$ the atomic form factor. The atomic form factor is equal to the Fourier Transform of the electron charge distri- 


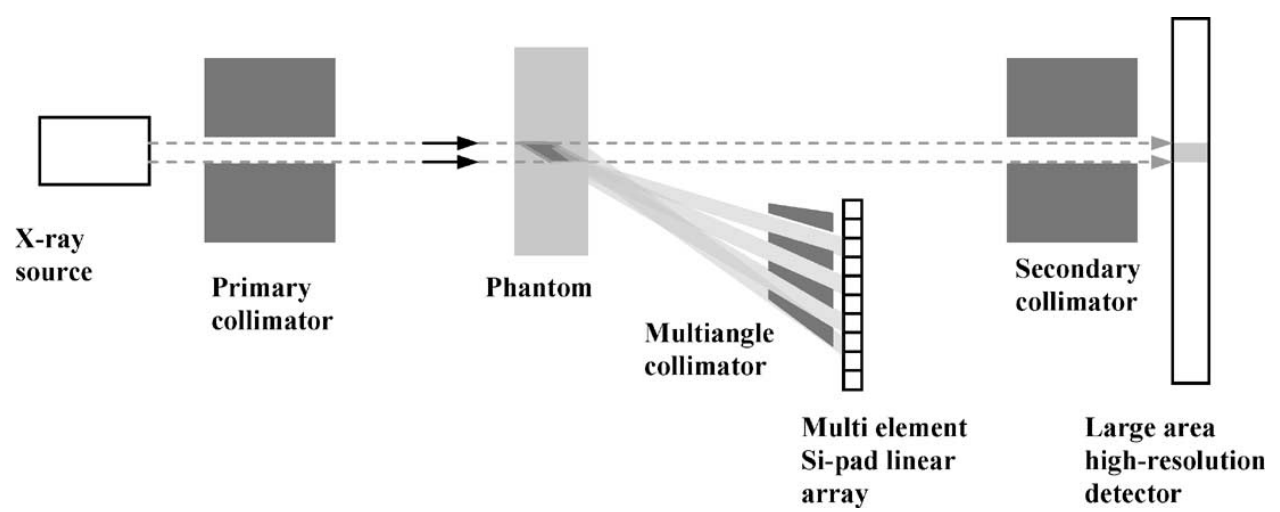

Fig. 1. Experimental set-up showing the concept of simultaneous measurements of the transmitted primary and the forward scattered X-rays from a phantom.

bution, which is a property of the material. For small values of $\theta$ it approaches $\mathrm{Z}$, while for large values of $\theta$ tends toward zero. However, the IAM does not include intramolecular or intermolecular effects caused by the close spacing of molecules in real materials. For compounds or mixtures, the molecular form factor is given by: $\mathrm{F}_{\mathrm{m}}^{2}(\chi) / \mathrm{W}=\sum\left(\mathrm{w}_{\mathrm{i}} / \mathrm{M}_{\mathrm{i}}\right) \mathrm{F}_{\mathrm{i}}^{2}\left(\chi, \mathrm{Z}_{\mathrm{i}}\right)$, where $\mathrm{w}_{\mathrm{i}}$ and $\mathrm{M}_{\mathrm{i}}$ are the mass fraction and the atomic mass of the $i$ th element and $\mathrm{W}$ the molecular weight. At low values of $\chi$, the diffraction from amorphous materials, such as most body tissues, is circularly symmetric around the primary beam. The scattering profiles show one or more broad peaks with an oscillatory behavior around the free-gas model, while toward higher values of $\chi$ the amplitude of the oscillation strongly decreases. As a final point, the differential cross section for a compound or a mixture can be expressed in terms of a linear differential scattering coefficient given by: $\mu_{\mathrm{s}}(\chi)=\mathrm{N}_{\mathrm{A}} \rho / \mathrm{M}\left\{\left(\mathrm{d} \sigma_{\mathrm{T}} / \mathrm{d} \Omega\right) \mathrm{F}_{\mathrm{m}}^{2}(\chi)+\left(\mathrm{d} \sigma_{\mathrm{KN}} / \mathrm{d} \Omega\right) \mathrm{S}_{\mathrm{m}}(\chi)\right\}$, where $\mathrm{N}_{\mathrm{A}}$ is the Avogadro number, $\mathrm{M}$ and $\rho$ are the molecular mass and the density of the material; the $\left(\mathrm{d} \sigma_{\mathrm{T}} / \mathrm{d} \Omega\right)$ and the $\left(\mathrm{d} \sigma_{\mathrm{KN}} / \mathrm{d} \Omega\right)$ are the Thomson and Klein-Nishina differential photon scattering cross sections, respectively; and $\mathrm{S}_{\mathrm{m}}(\chi)$ is the molecular incoherent scattering function, which can be calculated from the sum rule: $\mathrm{S}_{\mathrm{m}}(\chi) / \mathrm{W}=\sum\left(\mathrm{w}_{\mathrm{i}} / \mathrm{M}_{\mathrm{i}}\right) \mathrm{S}_{\mathrm{i}}(\chi)$, where $\mathrm{S}_{\mathrm{i}}(\chi)$ is the IAM scattering function.

The important parameters in the design consideration and optimization of X-ray imaging systems to clearly identify tissues of interest from other tissues surrounding them, and hence, to enhance the image contrast, are: the incident photon energy-scatter angle, the scatter intensity-tissue volume and the diffraction sensitivity-spatial resolution. By carefully incorporating these design factors, work is under way to build a laboratory prototype dual-sensor breast-imaging scanning system [11], which combines the diagnostic information from the conventional X-ray transmission with that from the low-angle X-ray scattering. Fig. 1 shows the experimental set-up and the concept for simultaneous measurements of the transmitted primary and the forward scattered X-rays using the same high-resolution micro-focus X-ray source. For the high-resolution $\mathrm{X}$-ray transmission system a large-area Hamamatsu CMOS (C7942) X-ray imager, with a pixel size of $50 \mu \mathrm{m}$ and a columnar structured $\mathrm{CsI}(\mathrm{Tl})$ scintillator directly deposited on its active area for high spatial resolution, is employed. Experiments carried out with the Contrast Detail
Mammography (CDMAM) phantom (type 3.2) were also showed that the CMOS sensor has high low-contrast sensitivity and small-detail visibility. It was able to resolve a range of small objects with a diameter between 0.16 and $3.2 \mathrm{~mm}$ corresponding to minimum detectable thickness of 1 to 0.06 $\mu \mathrm{m}$, respectively, at an entrance surface dose of $\sim 4 \mathrm{mGy}$ (these results are presented in detail in another publication under preparation). Most importantly, the CMOS sensor with its direct addressable pixel readout (random access) provides us with the capability of selective readout and, therefore, lends itself well for simultaneous measurements.

The requirements for the coherent scatter detector can be summarized, as follows: 1) need of energy-resolved sensor capable of giving us position information; 2) ability of direct detection of X-rays; 3 ) need of low-noise since the coherent scatter signal is weak comparatively to transmission signal; the scatter signal is typically three-four orders of magnitude lower than the transmission one; therefore, need of discrimination between signal and noise by setting up a threshold; 4) ability of operation at or near room temperature and 5) need to determine the optimum angular range (i.e., range in $\chi: \chi_{\min } \leq \chi \leq \chi_{\max }$ ) of detection of photons when trying to distinguish structures. To address these requirements the coherent X-ray scatter detection system required the design and development of a high-resistivity multielement two-dimensional (2-D) Si-pad array with a multichannel low-noise pulse processing front-end electronics chip and a new DAQ system. Results regarding the characterization and optimization of the detector-readout electronics-DAQ system and its performance to measure diffraction signatures of most commonly used breast-equivalent materials of interest are presented here.

\section{COHERENT X-RAY SCATter DeteCtion System AND ITS ELECTRONICS}

The Si-pad detectors are sufficiently quantum efficient ( $\sim 70 \%$ at $17 \mathrm{keV})$ and operate in spectroscopic pulse-counting mode. These detectors developed in collaboration with SINTEF, Norway were optimized to measure X-ray photons in the energy range of 12-45 keV, with a FWHM energy resolution of about $1-3 \mathrm{keV}$. There are 3 detector modules. Each detector module consists of an array of $6 \times 21$ pad detectors aligned on a 2 -mm pitch with a $12 \mathrm{~mm} \times 42 \mathrm{~mm}$ of active area. The 
size of the active electrode of each pad is typically $1.8 \mathrm{~mm}$ $\times 1.8 \mathrm{~mm}$. Each detector module consists of a dc-coupled single sided device fabricated from high-resistivity 1-mm-thick silicon. A multiguard ring structure was incorporated around the edge of each detector module to allow stable operation in full depletion, corresponding to operation bias voltages, $V_{\text {bias }}$, of 150-300 V. Thin metal traces from each detector pad lead to bond pads located on one side of the detector suitable for direct wire bonding to a PCB motherboard containing the XA1.3, a high-density IDE AS (Norway) readout chip. There are two bond pads per channel, each pair of pads on $100 \mu \mathrm{m}$ pitch. The bond pad dimensions are $120 \mu \mathrm{m} \times 75 \mu \mathrm{m}$. Biased guard ring electrodes surround each detector pad to minimize crosstalk.

The XA1.3 is a 128-channel pulse counting custom-tailored ASIC. It is a low-noise low-power self-triggered and data-driven charge signal acquisition chip, which upon a signal detection above the adjustable threshold, responds with sending out the energy- and position-information of the hit channel. A charge pulse collected by any of the 128 channels is amplified and filtered by the preamplifier and the shaper in the channel. If the amplitude of the peak analog signal is larger than the external adjustable threshold voltage $\mathrm{V}_{\text {th }}$, a trigger signal is generated internally and the trigger plus the channel address are shown as analog current outputs. Each detector module consists of an FR4 support card with a high-density 26-pin flat cable connector to the external DAQ circuit.

The DAQ system consists of a programmable gate array to control the data acquired from three 12-bit A/D converters, which are clocked continuously. When a strobe pulse occurs, the relevant simultaneous samples from the converters are packed into a 5-byte data package and clocked into a FIFO. These packages are sent contiguously into the FIFO as and when strobes demand it, one package per strobe. The FIFO output transmits data to a PC data acquisition card, NI (National Instruments, U.K.) PCI 6534 buffered digital I/O card. The maximum data rate into the NI card is $20 \mathrm{Mb} / \mathrm{s}$, by ACK/REQ handshake. The timing and control block splits time into integration periods, the duration of which is set by an external 8-bit port. Circuits to provide bias supplies and interface signals from/to the XA1.3 chips as required were also developed. Menu-driven DAQ software runs the whole detector-electronics system. All the configuration parameters are set through this software. The DAQ software operates either in a calibration or a normal mode. Before it can be used to acquire data (normal mode), the software needs to calibrate the detector and the ADC channels (calibration mode) and to produce look-up tables. This involves acquiring data for decoding the detector address from ADC channels B and C and the signal strength data from ADC channel A for five different calibration signal strengths. Once the calibration has been done, one can switch to normal mode, where data is acquired and automatically decoded. Gain shift correction and energy conversion in $\mathrm{keV}$ [according to: $\mathrm{E}(\mathrm{keV})=$ $\mathrm{V}(\mathrm{mV}) \times \mathrm{C}(\mathrm{pF}) \times \mathrm{W}(\mathrm{eV} /$ electron - hole pairs $) \times 6.25]$ are also implemented. As a final point, noisy and/or "dead" channels can be disabled from being able to trigger, so, they will not contribute in the noise of the system. Special LabView-based programme was developed for the analysis and the

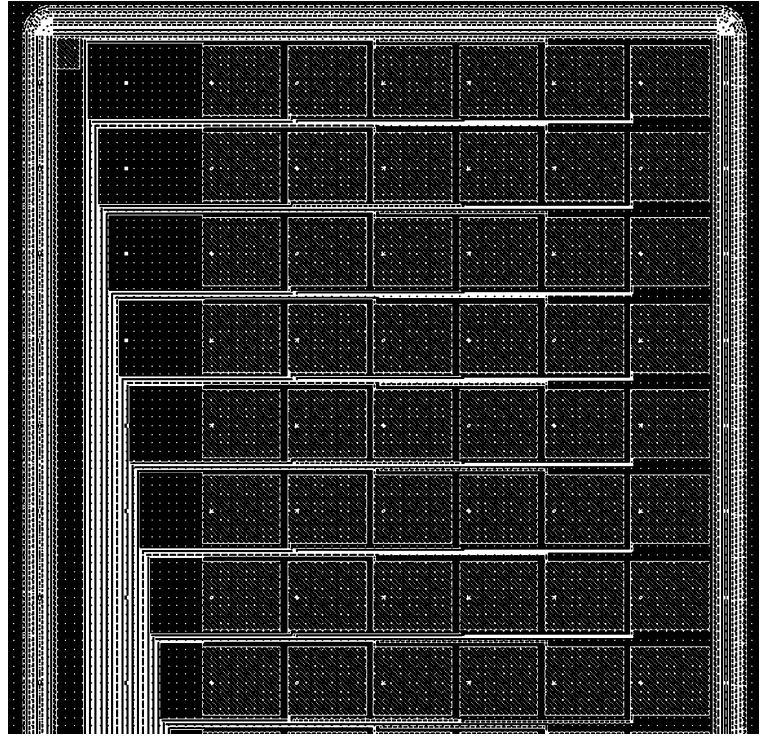

Fig. 2. Layout of the multielement Si-pad array.

visualization of the data-in raw ADC values and/or Energy in $\mathrm{keV}$ - The data can be presented in a single-channel spectral response and/or overall spectrum across all detector channels; hit map, mean amplitude, FWHM across all channels; 2-D intensity maps showing the pulse height for each channel; and finally, peak centroid and energy resolution measurements can be performed. Fig. 2 shows the internal layout of the multielement Si-pad array.

\section{Characterisation OF THE COHEREnt X-RAY SCATter DETECTION SYSTEM}

To begin, a set of calibration measurements was performed to optimize the working condition of the detection system. These tests were carried out by sending voltage pulses to the detector channels through the test input. Working in calibration mode, a low-noise precision pulser was used to give an absolute calibration of the multicell Si-pad array with a reasonable accuracy. Five calibration voltages, for better data averaging, were implemented with a threshold voltage of $V_{\text {th }}=0.1 \mathrm{mV}$. The bias voltage applied to the detector via the 710 Quad Bias Supply NIM unit was $V_{\text {bias }}=150 \mathrm{~V}$, while the leakage current for the detector module was $\mathrm{I}_{\mathrm{L}}=0.02 \mu \mathrm{A}$ given at any time by the same NIM unit. A calibration graph of the calibration voltages in $\mathrm{mV}$ versus the ADC centroid values was plotted and a linear regression was applied as shown in Fig. 3. It is clear that the front-end electronics chip provides good linearity to the detection system. The capabilities of the system were successfully tested for Vth as low as $0.07 \mathrm{mV}$ and calibration voltage as low as $0.5 \mathrm{mV}$. Once the detector was calibrated and the energy look-up tables were generated, the normal mode was switched on for the rest of the experiments.

Further, to determine the optimum working conditions with respect to the operating voltage $V_{\text {bias }}$, threshold voltage $V_{\text {th }}$, leakage current $\mathrm{I}_{\mathrm{L}}$, and temperature $\mathrm{T}$, as well as to assess the noise performance of the coherent scatter detection system as regards the operating conditions and the threshold set up for discrimination between scatter signal and noise, experiments were 


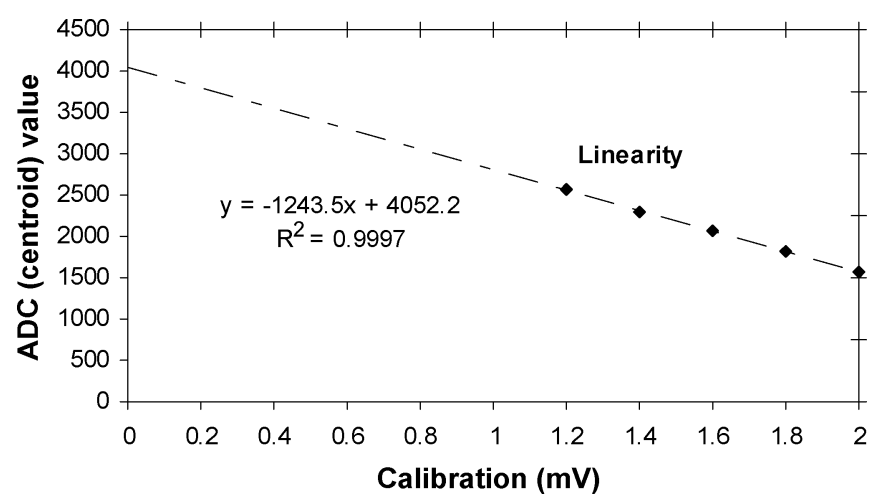

Fig. 3. Calibration graph of the multielement Si-pad array.

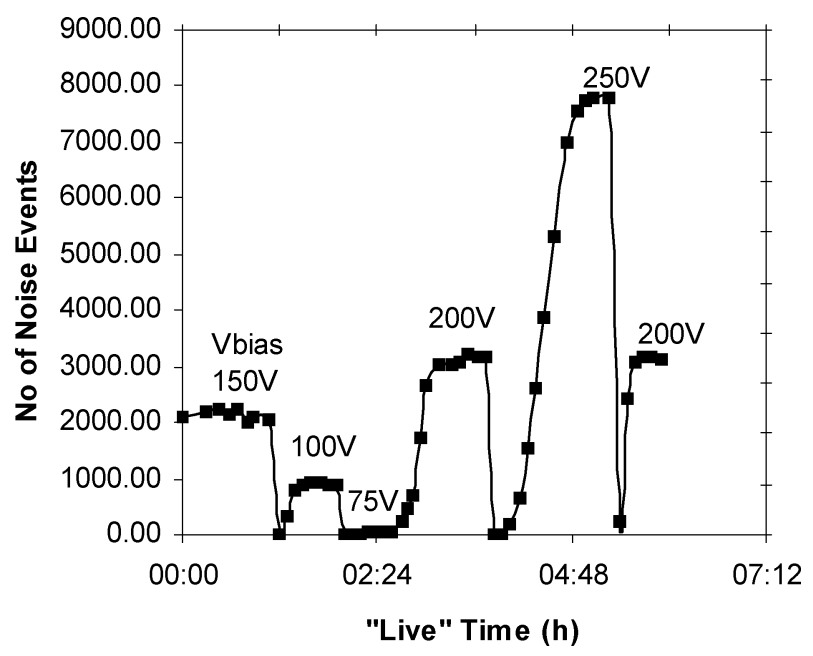

Fig. 4. Noise performance of the coherent scatter detection system for different operating voltages as given by the design specifications (as given in Section II). "Live" time represents the period since the Si-pad detector was turned on and reached equilibrium.

carried out to measure the number of noise events across the 126 channels of the multicell array without any signal from a pulser or a radiation source. Fig. 4 shows the noise behavior of the coherent scatter detection system for different operating voltages, as given by the design specifications (as described in Section II), at $V_{\text {th }}=0.07 \mathrm{mV}$, while Fig. 5 for different operating voltages and different threshold voltages.

Obviously, the number of noise events measured is low, in comparison with the number of events when using the source, for all the operating voltages given by the design specifications. Between the different operating voltages, there was a very short period of time while the detector was reaching equilibrium; then the noise events were increased up to the level of the corresponding detector bias value. It was decided for all future experiments to operate the Si-pad detector at $\mathrm{V}_{\text {bias }}=150 \mathrm{~V}$, which allows stable operation in full depletion and low-noise. By reducing the $V_{\text {th }}$ and/or increasing the $V_{\text {bias }}$, the number of noise events is increased. However, for $V_{\text {bias }}=150 \mathrm{~V}$ and in the $\mathrm{V}_{\text {th }}$ range of interest $(0.07-0.12 \mathrm{mV})$ the number of noise events is extremely low. For example, less than 100 noise events across the 126 detector channels for $\mathrm{V}_{\text {bias }}=150 \mathrm{~V}$ and $\mathrm{V}_{\text {th }}=$ $0.1 \mathrm{mV}$. This specific range of $\mathrm{V}_{\text {th }}$ allows clear distinction between the signal and the noise, as well as the detection of X-rays within the energy range of interest.

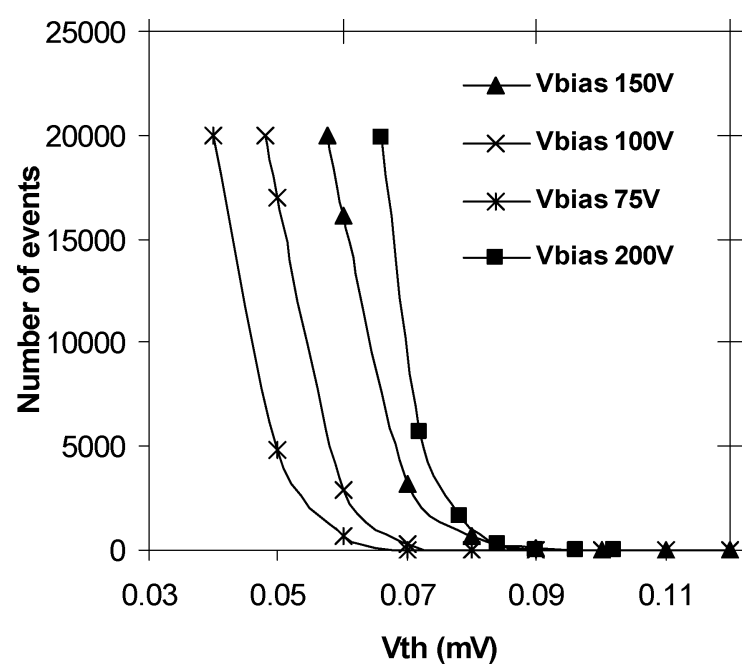

Fig. 5. Noise performance of the coherent scatter detection system for different operating voltages and different threshold voltages.
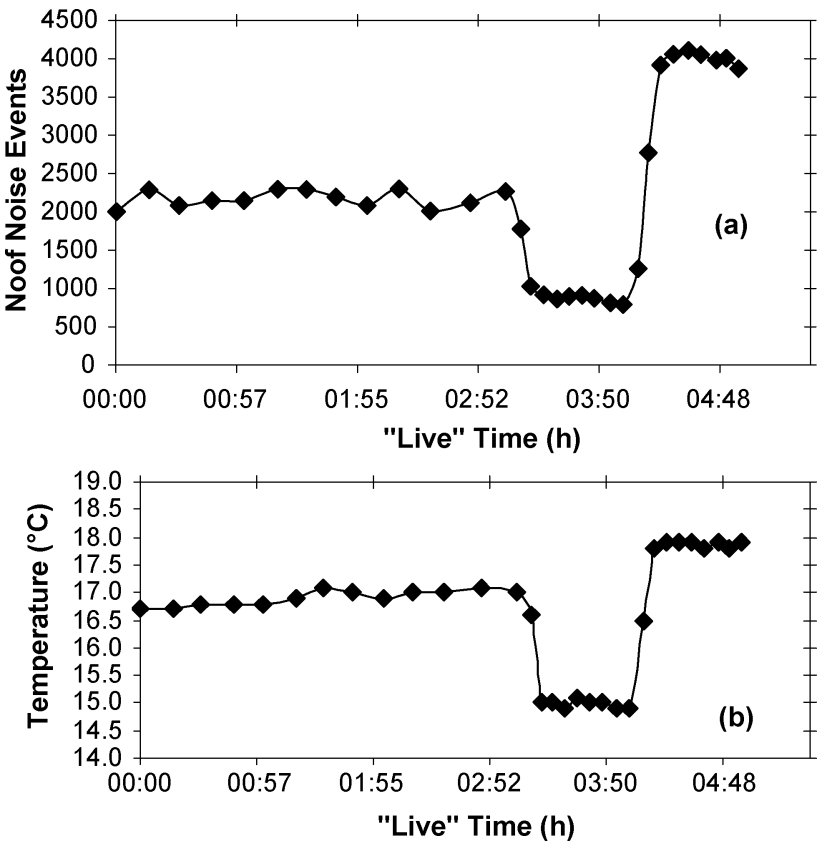

Fig. 6. (a) Noise performance of the coherent scatter detection system for different temperatures. (b) Variation of temperature during the "live" time of the experiment.

Since the XA1.3 readout chip is susceptible to small changes in the operating temperature and requires operating at stable temperature, a peltier cooling element was added at the exterior of the detector-electronics metal box, as well as a circuit was designed and built to regulate and measure the temperature inside and outside the metal box. Fig. 6 shows the noise behavior of the coherent scatter detection system for different temperatures, while Fig. 7 the distribution of noise events for different temperatures and leakage currents. Clearly, the number of noise events remains very low if the detection system operates at stable temperature, which is actually near the room temperature. For the range of temperatures of interest, i.e., at or near the room temperature, the leakage current of the Si-pad detector does not increase significantly, and hence, the number of noise events is very low. 


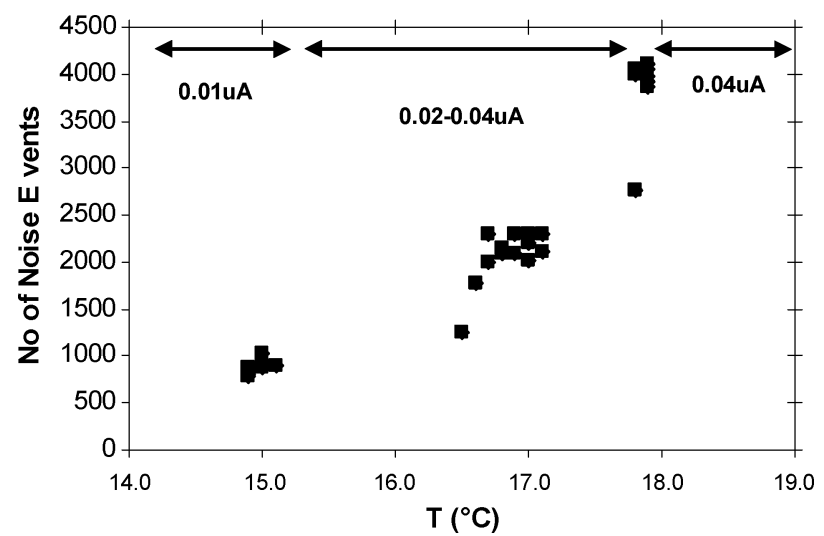

Fig. 7. Distribution of noise events for different temperatures and different leakage currents.

The next step was to assess and optimize the energy response of the Si-pad based low-angle scatter system within the energy range (12-45 keV) given in the design specifications and to determine its energy resolution by employing a laboratory variable energy X-ray source, Am-241, (358 MBq) to produce the X-rays of specific energies. This source consists of a compact assembly containing a sealed ceramic primary Am-241 source. It excites characteristic X-rays from six different targets mounted on a rotary holder. Each target can be presented to the primary source in turn and the characteristic X-rays from the target are emitted through a 4-mm diameter aperture. To cover the energy rage of interest, we made use of the following targets: Mo (17.44 and $19.63 \mathrm{keV}$ with relative intensities of 100 and 15 , respectively), $\mathrm{Ag}$ (22.10 and $24.99 \mathrm{keV}$ with relative intensities of 100 and 16, respectively), $\mathrm{Ba}$ (32.06 and $36.55 \mathrm{keV}$ with relative intensities of 100 and 18, respectively), and $\mathrm{Tb}$ (44.26 and $50.65 \mathrm{keV}$ with relative intensities of 100 and 20, respectively). The figures in parentheses correspond to $\mathrm{K}_{\alpha}$ and $\mathrm{K}_{\beta}$ lines, respectively. The experiments for the energy spectral response were performed at $\mathrm{V}_{\text {bias }}=150 \mathrm{~V}, \mathrm{I}_{\mathrm{L}}=0.02 \mu \mathrm{A}$ and $\mathrm{V}_{\text {th }}=0.1 \mathrm{mV}$. The energy response of the Si-pad array across all 126 channels and for different energies is shown in Fig. 8 (where event denotes number of total counts across the 126 channels). The DAQ system was initially designed in a way that the ADC scale is in the opposite direction of energy, i.e., the $\mathrm{E}=0 \mathrm{keV}$ corresponds to $\mathrm{ADC}=4095$. The FWHM energy resolution measured for Mo and $\mathrm{Tb}$ is $3 \mathrm{keV}$ for all channels and $1 \mathrm{keV}$ for single channel. Apparently, the multielement Si-pad detector can detect X-rays in the energy range of 17-45 $\mathrm{keV}$ and can resolve the $\mathrm{K}_{\alpha}$ and $\mathrm{K}_{\beta}$ peaks of $\mathrm{Tb}, \mathrm{Ba}, \mathrm{Ag}$, and Mo, which tie in with the known branching ratios, as given above. The detection efficiency of the $\mathrm{Si}$-pad detector varies between $\sim 65 \%$ at $20 \mathrm{keV}$ to $\sim 17 \%$ at 45 $\mathrm{keV}$.

To quantitatively assess the low-angle X-ray scatter performance of the special-purpose designed multielement Si-pad arrays, the scattering profiles of water, some most commonly used breast-equivalent materials (PMMA, nylon, polyethylene) and breast adipose tissue (from mastectomy) were measured. A high-resolution micro-focus Mo source operating at $30 \mathrm{kVp}$ was used. An absorption filter of $\mathrm{Zr}$ was placed in front of the $\mathrm{X}$-ray tube to monochromate the $\mathrm{X}$-rays from the continuum to an almost $17.44 \mathrm{keV}$ peak (the average energy of mammog-

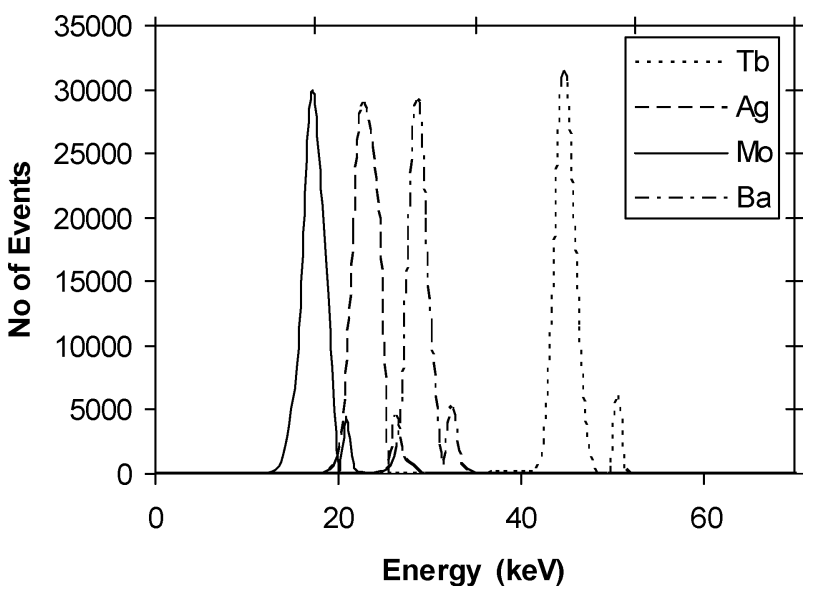

Fig. 8. Spectral response of the multicell Si-pad array to different X-ray energies of interest.

raphy). An appropriate arrangement of slits was led to a highly collimated beam incident on the samples, which were placed on a rotating table in the centre of the scattering geometry to ensure that different parts of the specimens were equally exposed to $\mathrm{X}$-rays. Another set of collimators was placed in front of the Si-pad detectors to make sure that the well-resolved coherently scatter patterns at a fixed angle of $6^{\circ}[12]$ were obtained. The thickness of each sample was chosen according to the inverse of attenuation coefficient $1 / \mu$ to provide adequate number of single-scattering events while minimizing the probability of multiple scattering; a polypropylene cylindrical container with a wall thickness of $0.1 \mathrm{~mm}$ to minimize the effects of attenuation was employed as a sample holder. The measured scattering profiles in terms of linear scattering coefficient per unit solid angle $\mu_{\mathrm{s}}(\chi)$ (as defined in Section I) for the aforementioned materials are shown in Fig. 9. As part of the experimental data analysis, each measured scattering signature was subjected to corrections for scatter and attenuation and normalized with the theoretical data in the region of momentum transfer $\chi$ where no interference effects are expected.

The scattering profiles measured show one predominant peak, or several as in the case of polyethylene and nylon, where interference effects are most important and its position is characteristic of the material; then the intensity falls in an oscillatory way according to the free-gas model (IAM). These results are in excellent agreement with former published data [4], [6], [7], and [10] regarding the peak position ( $\chi$ value) and the shape (broad or sharp). The peak position for the materials used are: $0.78 \pm 0.02 \mathrm{~nm}^{-1}$ for PMMA; $1.10 \pm 0.02 \mathrm{~nm}^{-1}$ for breast adipose tissue; $0.90 \pm 0.02 \mathrm{~nm}^{-1}$ and $1.40 \pm 0.02 \mathrm{~nm}^{-1}$ for polyethylene; $1.20 \pm 0.02 \mathrm{~nm}^{-1}$ and $1.40 \pm 0.02 \mathrm{~nm}^{-1}$ for nylon; and $1.60 \pm 0.02 \mathrm{~nm}^{-1}$ for water. However, there is a substantial difference in the relative magnitudes of the peaks, i.e., in the main peak height, defined as the ratio of the value of the linear differential coefficient at the main peak position to the average of the values corresponding at the region of momentum transfer values which is independent of interference effects. The difference arises because surrounding irradiated background sources (e.g., sample synthesis, preparation and geometry, sample container, etc. ...) contribute additional scatter to the measured $\mathrm{X}$-ray coherent scatter signal. Also, the attenuation of the pri- 

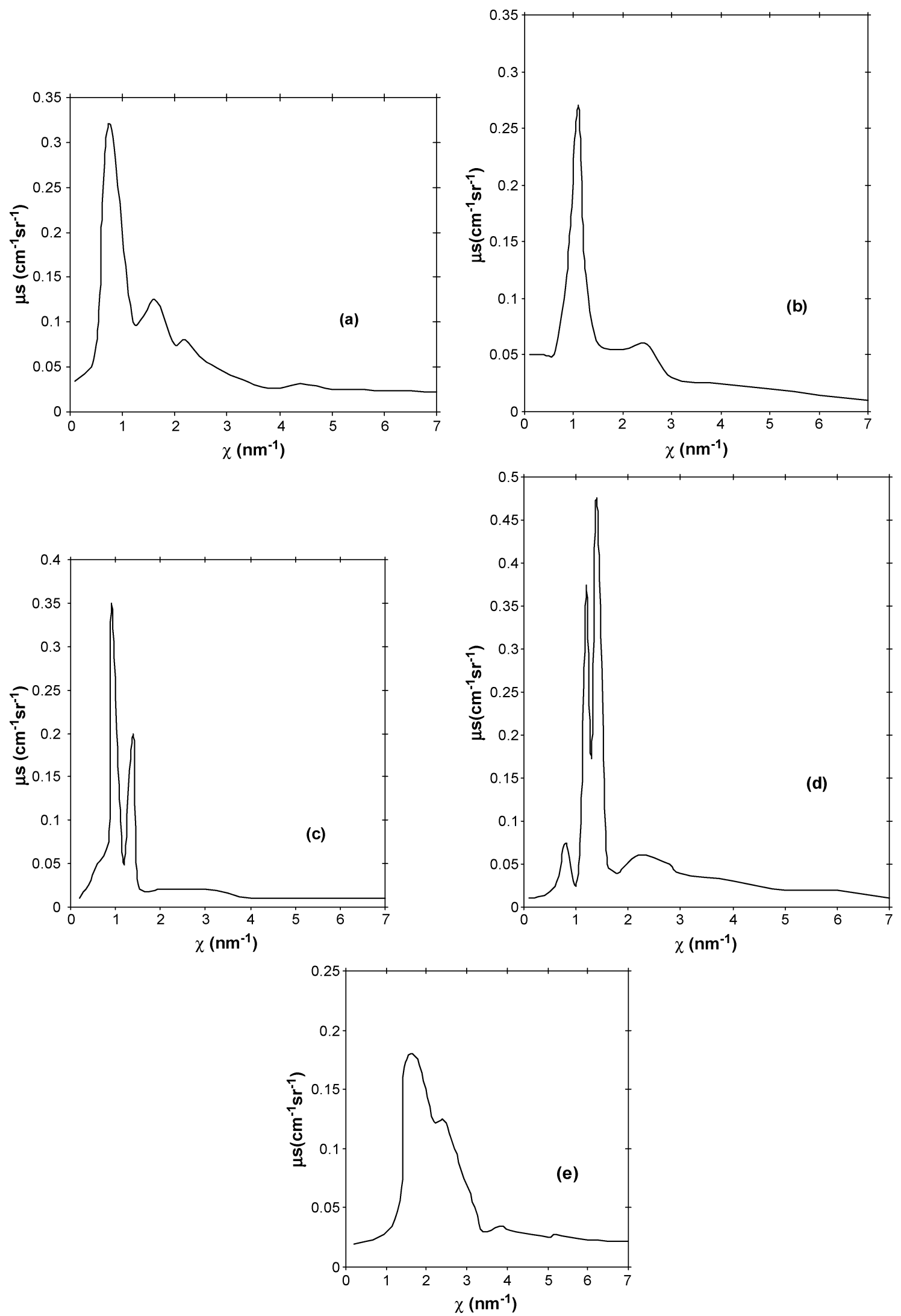

Fig. 9. Measured scattering profiles of (a) PMMA. (b) Breast adipose tissue. (c) Polyethylene. (d) nylon. (e) Water using the Si-pad coherent scatter detection system.

mary and scattered beams depends on the depth of the scattering volume element within the sample, and, therefore, modifies the scattering signatures for a given material. Further, the accuracy of the scattering volume localization, as well as the height of the 
measured diffraction peaks are shaped and determined by the dimensions of the system and several experimental parameters, such as the voltage and current of the X-ray source, the scattering angle, the primary beam diameter and the dimensions of the scatter collimator. Finally, the contribution of multiple scattering should be investigated. All these issues should be thoroughly examined and addressed before we proceed with the implementation of low-angle scatter imaging in the detection of breast cancer, since the various breast tissues are bathed in fluids such as blood and any lesion is typically surrounded by benign and other type tissues that can produce scatter signals, which will be difficult, if not impossible, to separate and localize independently from the small signal produced by malignancy.

\section{SUMMARY}

A coherent X-ray scatter detection system based on a high-resistivity multielement 2-D Si-pad array, a multichannel low-noise pulse processing front-end electronics chip, the XA1.3, and a new DAQ system was designed and developed. It was shown that this novel detection system meets the design specifications combining good energy resolution (1-3 keV) with position information; very good spectral response (17-45 $\mathrm{keV}$ ) to the X-ray energies of interest; excellent low-angle $\mathrm{X}$-ray scatter performance with highly resolved diffraction signatures at mammographic energies; low-noise behavior; and operates at near room temperature without the need of cryogenic cooling. The multielement 2-D Si array in combination with a suitable multiangle collimator provides us with the capability of applying a mixture of angle and energy dispersion techniques and has the potential to collect multiple scatter signals simultaneously, either from the same voxel or from a large number of voxels, to maximize the content of diagnostic information at shorter measurement scanning times. It is, therefore, demonstrated that this Si-pad based coherent scatter detection system lends itself well to the detection of diffraction signatures and its implementation to a dual-sensor X-ray breast imaging system that will combine transmission and coherent scattering procedures.

\section{ACKNOWLEDGMENT}

The authors would like to thank D. Harmer and J. Hearne for their valuable contribution to the project.

\section{REFERENCES}

[1] R. Wilson, private communication, 2003.

[2] P. C. Johns and M. J. Yaffe, "Coherent scatter in diagnostic radiology," Med. Phys., vol. 10, no. 1, pp. 40-50, Jan./Feb. 1983.

[3] R. J. Leclair and P. C. Johns, "X-ray forward scatter imaging: experimental validation of model," Med. Phys., vol. 28, no. 2, pp. 210-219, Feb. 2001.

[4] J. Kosanetzky, B. Knoerr, G. Harding, and U. Neitzel, "X-ray diffraction measurements of some plastic materials and body tissues," Med. Phys., vol. 14, no. 4, pp. 526-532, July/Aug. 1987.

[5] S. H. Evans, D. A. Bradley, D. R. Dance, J. E. Bateman, and C. H. Jones, "Measurements of small-angle photon scattering for some breast tissues and tissue substitute materials," Phys. Med. Biol., vol. 36, pp. 7-18, 1991 .

[6] A. Tartari, E. Casnati, C. Bonifazzi, and C. Baraldi, "Molecular differential cross sections for X-ray coherent scattering in fat and polymethyl methacrylate," Phys. Med. Biol., vol. 42, pp. 2551-2560, 1997.

[7] D. E. Peplow and K. Verghese, "Measured molecular coherent scattering form factors of animal tissues, plastics and human breast tissue," Phys. Med. Biol., vol. 43, pp. 2431-2452, 1998.

[8] G. Kidane, R. D. Speller, G. J. Royle, and A. M. Hanby, "X-ray scatter signatures for normal and neoplastic breast tissues," Phys. Med. Biol., vol. 44, pp. 1791-1802, 1999.

[9] R. A. Lewis, K. D. Rogers, C. J. Hall, E. Towns-Andrews, S. Slawson, and A. Evans et al., "Breast cancer diagnosis using scattered X-rays," J. Synchr. Rad., vol. 7, pp. 348-352, 2000.

[10] M. E. Poletti, O. D. Goncalves, and I. Mazzaro, "X-ray scattering from human breast tissues and breast equivalent materials," Phys. Med. Biol., vol. 47, pp. 47-63, 2002.

[11] D. G. Darambara, R. D. Speller, and P. J. Sellin, "Development of a dual detector system based on a-Si:H arrays and multi-element silicon detectors for diffraction enhanced breast imaging," IEEE Trans. Nucl. Sci., vol. 49, pp. 1012-1016, June 2002.

[12] E. P. Muntz, T. Fewell, R. Jennings, and H. Bernstein, "On the significance of very small angle scattered radiation to radiographic imaging at low energies," Med. Phys., vol. 10, no. 6, pp. 819-823, Nov./Dec. 1983. 